\title{
Bonding of CO to Metal Particles: Photoelectron Spectra of $\mathrm{Ni}_{n}(\mathrm{CO})_{m}{ }^{-}$and $\mathrm{Pt}_{n}(\mathrm{CO})_{m}{ }^{-}$Clusters
}

\author{
G. Schulze Icking-Konert, H. Handschuh, G. Ganteför, and W. Eberhardt \\ Institut für Festkörperforschung, Forschungszentrum Jülich, 52425 Jülich, Germany
}

\begin{abstract}
Photoelectron spectra of mass-selected negatively charged bare $\mathrm{Ni}_{n}{ }^{-}$and $\mathrm{Pt}_{n}{ }^{-}$clusters are compared with the spectra of $\mathrm{Ni}_{n}(\mathrm{CO})_{m}{ }^{-}$and $\mathrm{Pt}_{n}(\mathrm{CO})_{m}{ }^{-}$clusters. The spectra indicate that saturation with $\mathrm{CO}$ is caused by the formation of an electronic closed shell and not due to steric hindrance as on a surface, and that the strength of the $\pi$ backdonation of the $\mathrm{CO}$ chemisorption bond to a small particle is stronger than to a surface. The antibonding metal $\left(d_{z^{2}}-5 \sigma\right)$ orbital resulting from the interaction of the CO $5 \sigma$ with the metal $d_{z^{2}}$ orbitals is directly observed for $\mathrm{Ni}_{1}(\mathrm{CO})_{3}{ }^{-}$.

PACS numbers: $36.40 . \mathrm{Mr}, 73.20 . \mathrm{Hb}, 82.65 . \mathrm{My}$
\end{abstract}

The question of the nature of the chemisorption bond of $\mathrm{CO}$ to transition metal atoms connects two large field in basic research: carbonyl chemistry and surface science. It is now well accepted that the bonding can be described by the $\sigma$-donation and $\pi$-backdonation scheme (Blyholder model [1]). The CO $5 \sigma$ orbital interacts with the metal $\sigma$ orbitals (donation) and the metal $d_{\pi}$ orbitals form a bond with the unoccupied $\mathrm{CO} 2 \pi$ orbitals (backdonation). This model has been verifie by many experimental studies using techniques such as photoelectron and vibrational spectroscopy.

One property of carbonyls that has no direct analog in surface science is the following: the stability of the complex due to an uptake of a certain number $m$ of COs (saturation). Carbonyls with a lower number of ligands are "unstable." According to Lauher's electron counting rules [2-4] the stability is explained by the formation of an electronic closed shell with a "magic" number of electrons [e.g., 18 electrons in $\left.\mathrm{Ni}(\mathrm{CO})_{4}\right]$. Therefore, the stabilization of a carbonyl is caused by a delocalized electronic interaction between the ligands and the metal core. In contrast, the saturation of $\mathrm{N}_{2}$ (which has a similar total number of valence electrons as $\mathrm{CO}$ ) on $\mathrm{Ni}_{n}{ }^{-}$and $\mathrm{Pt}_{n}{ }^{-}$ clusters is achieved at $m \approx n[5]$ and may be described by a topological approach suggesting a strong preference for "top" sites [6]. On a surface, saturation with CO is caused by steric hindrance between neighboring COs. We here present photoemission studies of reacted mass selected clusters of the type $M_{n}(\mathrm{CO})_{m}{ }^{-}$varying $m$ continuously from zero up to saturation. Our data indicate the formation of a closed electronic shell at saturation corresponding to a change in the metal $(\mathrm{Ni}, \mathrm{Pt})$ atom configuratio from $d^{9} s$ to $d^{10}$.

So far, there is an agreement that the $\mathrm{CO}$ chemisorption bonds to a surface and to a cluster are similar [7]. The observed frequencies of the $\mathrm{CO}$ stretch vibration, which alters with the strength of the backdonation interaction, are similar for carbonyls and for $\mathrm{CO}$ molecules bound in a corresponding site on a surface [8]. This similarity is also the basic assumption of cluster model calculations, a theoreti- cal method taking a small fragment of a surface instead of an infinit real surface for modeling surface data. One important question, however, has not been addressed by these calculations: Is the well-known catalytic activity of small particles due to topologic or electronic effects? If electronic effects play a role then a difference reflecte by the vibrational frequencies is expected for the chemisorption in carbonyls, clusters, and surfaces.

Concerning the similarity of the $\mathrm{CO}$ stretch frequencies, in both surfaces [9] and carbonyls [10] one has to take the coverage dependence into account. In general, the stronger the bond the lower the frequency because of the increased $\pi$ backdonation. This trend is counteracted by the dipole interaction in the adsorbate which causes a frequency shift to higher frequencies with increased coverage. The coverage in carbonyls (about 2-4 CO molecules per metal atom [4] is at least twice that of surfaces, and possibly the chemisorption bond in small particles is stronger than on a surface. Because of higher coverage, the measured frequencies are increased to approximately the values of the surface species. Therefore, we perform an experiment measuring the $\mathrm{CO}$ stretch vibration on metal clusters with a low CO coverage, i.e., unsaturated carbonyls of the type $M_{n}(\mathrm{CO})$. We fin frequencies lower than on the corresponding surfaces indicating a stronger backdonation interaction. This interaction weakens the $\mathrm{CO}$ bond, the key step in a catalytic reaction involving $\mathrm{CO}$.

Photoelectron spectroscopy of mass-separated, negatively charged clusters [11] allows the study of all possible combinations of metal atoms and ligands far beyond the experiments restricted to stable carbonyls or their fragments $[12,13]$. Negatively charged clusters are directly produced by a standard laser vaporization source [11]. Within the high pressure region of the extender a variable amount of $\mathrm{CO}$ can be added to the helium seeding gas. After passing the skimmer, the cluster anions are accelerated in a pulsed electric field Depending on their time of fligh (TOF) the clusters separate into a chain of bunches of define cluster size. The kinetic energy of electrons from a selected bunch, which are detached by a UV laser 


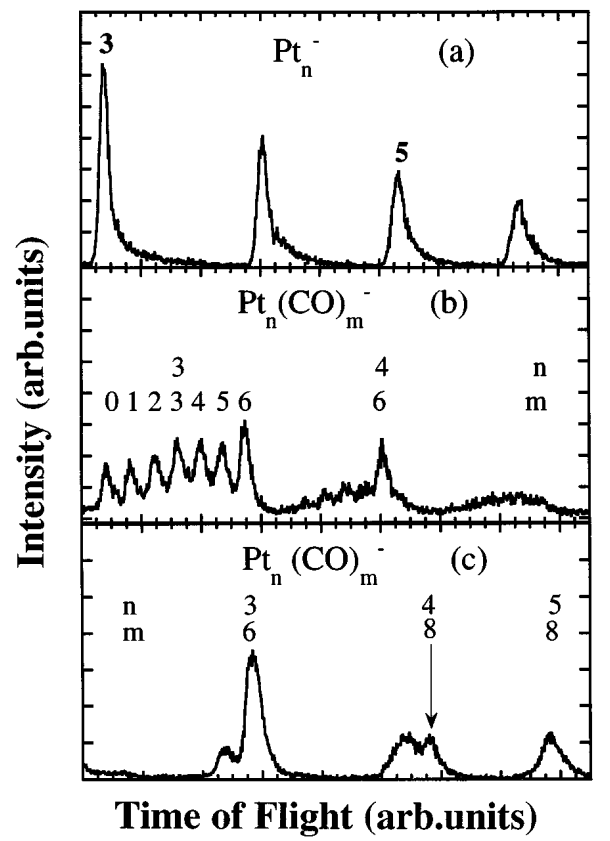

FIG. 1. Section of the time-of-fligh mass spectrum of (a) bare and reacted $[(\mathrm{b})$ medium amount of $\mathrm{CO}$; (c) high amount of $\mathrm{CO}] \mathrm{Pt}_{n}{ }^{-}$clusters generated in a laser vaporization source.

pulse, is measured using a "magnetic bottle" TOF electron spectrometer [11].

Figure 1 compares TOF mass spectra of bare (a) and reacted $\mathrm{Pt}_{n}{ }^{-}$clusters (b),(c) for $n=3-6$. The intense features in the spectrum of the bare clusters are assigned to $\mathrm{Pt}_{n}{ }^{-}$for $n=3-6$. In Fig. 1(b) the $\mathrm{Pt}_{n}{ }^{-}$clusters have reacted with $\mathrm{CO}$ at intermediate concentration. Reaction products with a varying number of adsorbed $\mathrm{CO}$ molecules are observed for all clusters; e.g., in the case of $\mathrm{Pt}_{3}(\mathrm{CO})_{m}{ }^{-} m$ varies between $m=0$ and 6. In Fig. 1(c) the clusters have reacted with the maximum amount of $\mathrm{CO}$ attainable in our experiment. Now the saturated carbonyl anion $\mathrm{Pt}_{3}(\mathrm{CO})_{6}{ }^{-}$is the most intense peak of the $\mathrm{Pt}_{3}(\mathrm{CO})_{m}{ }^{-}$series in agreement with recent quantitative studies of the reaction rates of $\mathrm{Pt}_{n}{ }^{-}$clusters with $\mathrm{CO}$ [4].

In principle, photoelectron spectra can be recorded for all cluster anions observed in Figs. 1(a)-1(c) and we show spectra of $\mathrm{Pt}_{3}(\mathrm{CO})_{m}{ }^{-}(m=0,1,5,6)$ and $\mathrm{Pt}_{4}(\mathrm{CO})_{m}{ }^{-}$ $(m=0,1,7,8)$ in Figs. 2(a)-2(h). The spectra of the reacted clusters do not exhibit pronounced structures except for those of the saturated carbonyls $\left[\mathrm{Pt}_{3}(\mathrm{CO})_{6}{ }^{-}\right.$, $\mathrm{Pt}_{4}(\mathrm{CO})_{8}{ }^{-}$, where an intense peak $A$ is observed close to the emission threshold at lowest binding energy (BE) [Figs. 2(d) and 2(h)].

We will try to reconcile this experimental findin using the framework of the Blyholder model [1]. The chemisorption bond of $M_{n}(\mathrm{CO})_{m}$ can be described in a firs approximation by two main interactions: (i) the interaction of the occupied $5 \sigma$ orbitals of CO with the metal $\sigma$ orbitals (= metal $s / p$ and metal $d_{z^{2}}$ and (ii) the interaction of the unoccupied $2 \pi$ orbitals of $\mathrm{CO}$ with those derived

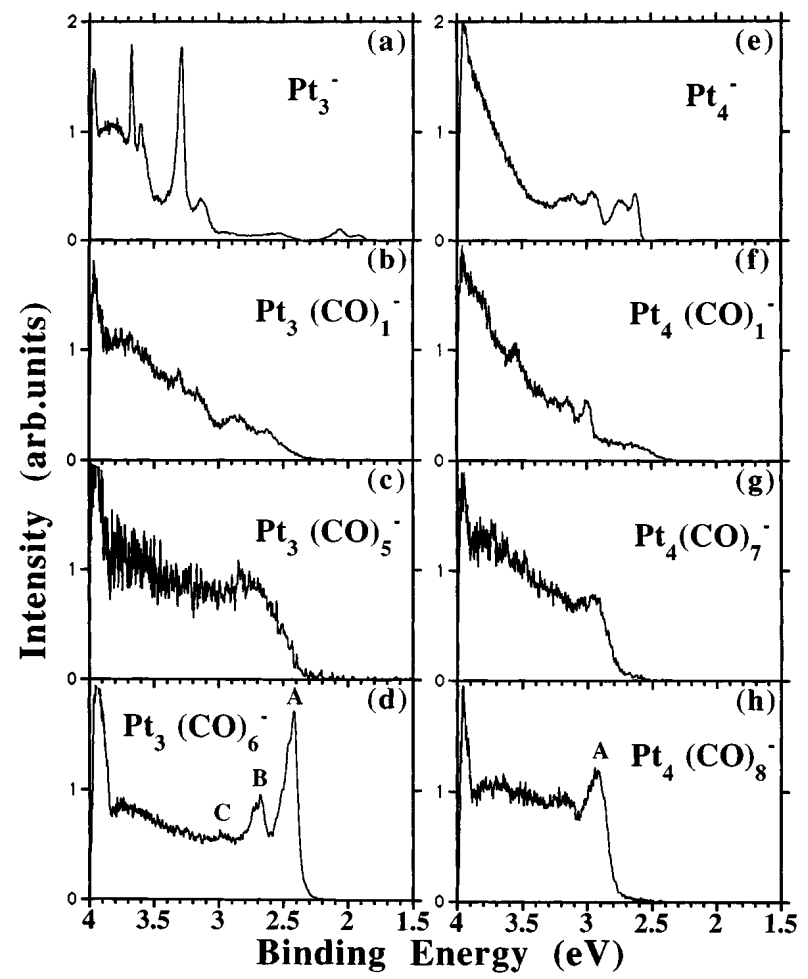

FIG. 2. Selected photoelectron spectra of $\mathrm{Pt}_{3,4}(\mathrm{CO})_{m}{ }^{-}$clusters. The photon energy is $h \nu=4.0 \mathrm{eV}$.

from metal $d_{\pi}$. The $\sigma$ interaction results in the stabilization of the $5 \sigma$ orbitals and a corresponding shift towards lower BE of the metal $s / p$ and $d_{z^{2}}$ orbitals (charge donation). The $\pi$ interaction stabilizes part of the metal $d$ manifold of states, while the CO $2 \pi$ orbitals are destabilized. The metal $d_{\pi}$ orbitals hybridize partially with the $\mathrm{CO} 2 \pi$ orbitals resulting in a "deformation" of these occupied orbitals towards the direction of the $\mathrm{CO}$ molecule. This corresponds to a net charge transfer to the $\mathrm{CO}$ molecule (backdonation). Because the deformation has a symmetry similar to the one of the antibonding $2 \pi$ orbitals the $\mathrm{C}-\mathrm{O}$ bond is weakened and the $\mathrm{CO}$ stretch vibration frequency is lowered.

The interaction between the $\mathrm{CO} 5 \sigma$ and the metal $s / p$ orbitals is strongly repulsive $[1,14,15]$. For $\mathrm{Ni}$ and $\mathrm{Pt}$, the metal $s / p$ orbitals are destabilized with an increasing number of ligands until they are completely unoccupied [1]. The ten metal valence electrons per atom occupy now $d$-derived states corresponding to a $d^{10}$ closed shell configuratio (with an admixture of $\mathrm{CO} \pi$ character). This explains the appearance of peak $A$ in Figs. 2(d) and 2(h). The unsaturated clusters have unoccupied $d$-derived orbitals, which can accommodate the additional electron of the anion. The featureless smooth emission signal can be assigned to photoemission from the $d$-derived manifold of states. In the saturated clusters the additional electron must occupy an orbital which is located above the manifold of $d$ states. This is probably the lowest of the CO $2 \pi$ derived orbitals for the following reasons: (i) the emission 
from this orbital exhibits a larger cross section because of its $\pi$ symmetry [16] explaining the relatively high intensity of the feature; (ii) emission from the $\mathrm{CO} 2 \pi$ orbital results in the excitation of the $\mathrm{CO}$ stretch vibration, because the $\mathrm{C}-\mathrm{O}$ equilibrium distance is altered by a change of the occupation of this orbital.

This explains the observation of the vibrational fin structure in the high resolution spectrum of $\mathrm{Pt}_{3}(\mathrm{CO})_{6}{ }^{-}$ [Fig. 3(a)] with a frequency of $2020 \pm 60 \mathrm{~cm}^{-1}$ [17],

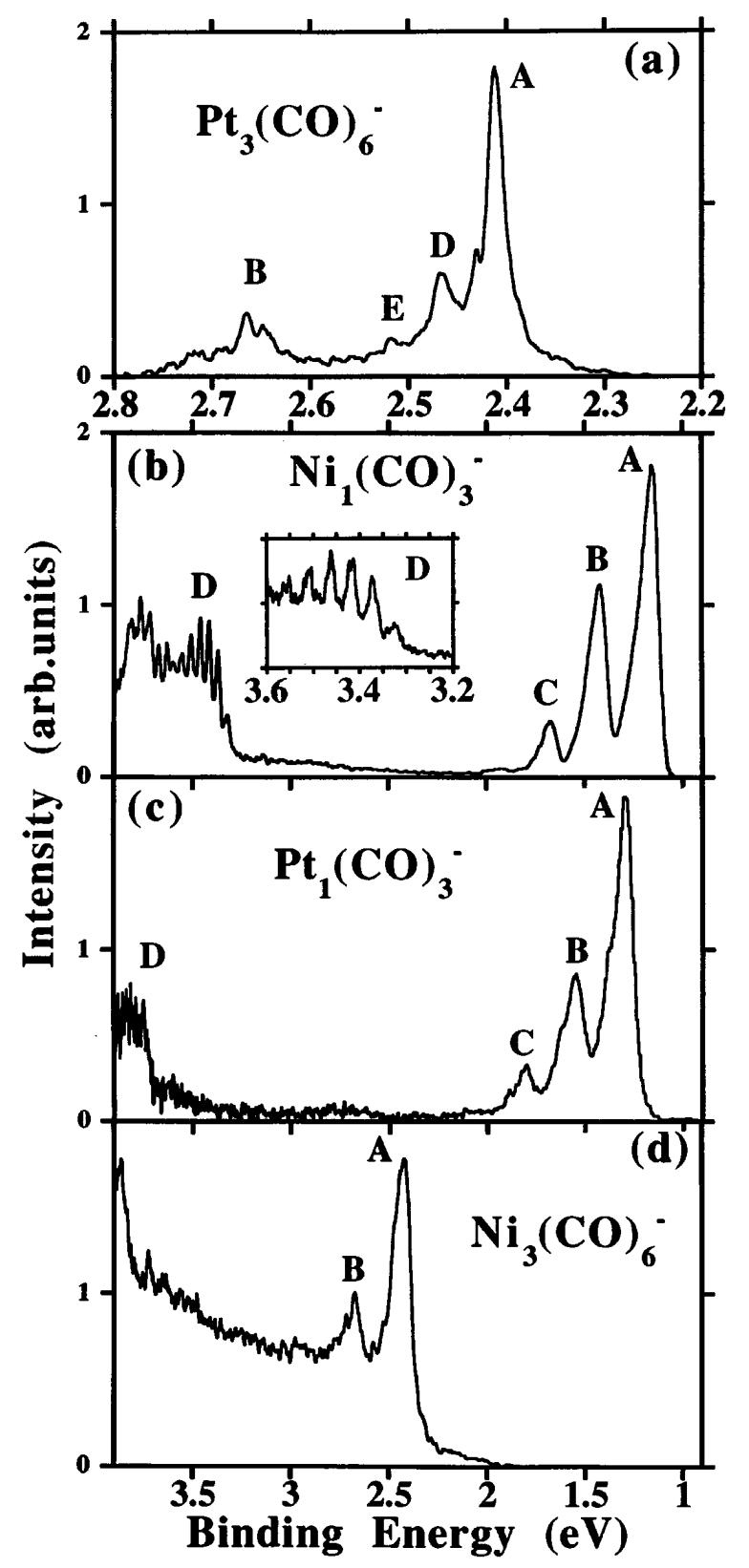

FIG. 3. Vibrationally resolved photoelectron spectra of (a) $\mathrm{Pt}_{3}(\mathrm{CO})_{6}{ }^{-}$, (b) $\mathrm{Ni}_{1}(\mathrm{CO})_{3}{ }^{-}$, (c) $\mathrm{Pt}_{1}(\mathrm{CO})_{3}{ }^{-}$, and (d) $\mathrm{Ni}_{3}(\mathrm{CO})_{6}{ }^{-}$. The photon energies are $h \nu=3.0,4.0,4.0$, and $4.0 \mathrm{eV}$, respectively. The peaks marked $A$ and $B$ in (a) correspond to the ones displayed in Fig. 2(d). The inset in (b) displays an expanded view of feature $D$. which is similar to the stretch frequency of $\mathrm{CO}$ adsorbed in a top site on a Pt surface (2065 $\mathrm{cm}^{-1}$ [9]). Our finding are in agreement with the calculations on $\mathrm{Pt}_{3}(\mathrm{CO})_{6}{ }^{2-}$ from Bullett [18], who found the uppermost 15 occupied molecular orbitals to be of metal $d$ character and (in the neutral unoccupied) an orbital at slightly higher energy with mainly CO $2 \pi$ character. The second low energy mode with $425 \pm 30 \mathrm{~cm}^{-1}$ [Fig. 3(a)] [17] is similar to metal-CO vibrations found on surfaces [19].

The similarity of the spectra of $\mathrm{Ni}_{1}(\mathrm{CO})_{3}{ }^{-}$and $\mathrm{Pt}_{1}(\mathrm{CO})_{3}{ }^{-}$[Figs. 3(b) and 3(c)] supports the assumption that the metal $s$ orbitals in the saturated species are unoccupied [1]. The spectra of $\mathrm{Ni}_{3}(\mathrm{CO})_{6}{ }^{-}$[Fig. 3(d)] and $\mathrm{Pt}_{3}(\mathrm{CO})_{6}{ }^{-}$[Fig. 2(d)] are also almost identical. The BEs of the atomic $\mathrm{Ni} 4 s$ and $\mathrm{Pt} 6 s$ orbitals differ by approximately $1 \mathrm{eV}$. If these orbitals would contribute significantl to the occupied orbitals in these carbonyls, the spectra would exhibit considerable differences. The observed CO stretch vibrations of $2090 \pm 80$ and $2060 \pm 80 \mathrm{~cm}^{-1}[A-B-C$ in Figs. 3(b) and 3(c), respectively] agree well with the corresponding surface data (Pt: $2065-2104 \mathrm{~cm}^{-1}$; Ni: $2045-2069 \mathrm{~cm}^{-1}$ [8,9]). We refrain from a discussion of the corresponding adsorption sites, since in a recent experiment [20] the simple relation between the $\mathrm{CO}$ stretch vibration and the adsorption site has been questioned.

For the unsaturated species $\mathrm{Ni}_{2}(\mathrm{CO})^{-}$and $\mathrm{Ni}_{3}(\mathrm{CO})^{-}$ we determine the frequency of the $\mathrm{CO}$ stretch vibration of the neutral to be $1800 \pm 80$ and $1750 \pm 80 \mathrm{~cm}^{-1}$, respectively [21]. These two values are lower than any such frequencies measured on Ni surfaces [22]. The large shifts indicate a stronger $\pi$ backdonation for a small cluster compared to a surface. In a recent measurement of the binding energy of $\mathrm{CO}$ to a $\mathrm{Pt}_{3}{ }^{-}$cluster an energy of about twice the surface value has been found [23]. Our photoelectron spectra indicate a large similarity of $\mathrm{Ni}$ and $\mathrm{Pt}$ carbonyls and we expect a correspondingly high binding energy for $\mathrm{CO}$ bound to a $\mathrm{Ni}_{3}{ }^{-}$cluster. Therefore, the observations of a high binding energy $\left(\mathrm{Pt}_{3}{ }^{-}\right)$and a low $\mathrm{CO}$ stretch frequency $\left(\mathrm{Ni}_{2}, \mathrm{Ni}_{3}\right)$ both indicate a stronger interaction for small clusters compared to surfaces. The reason for the stronger backdonation is probably related to the different electronic structure of the clusters compared to the bulk, because it is difficul to explain the experimental results based on geometric considerations only.

One basic experimental observation supporting the Blyholder model [1] is the stabilization of the CO $5 \sigma$ orbitals due to chemisorption observed in photoemission studies [24]. The $5 \sigma / d_{z^{2}}$ interaction should result in a destabilization of metal $d_{z^{2}}$ orbitals forming antibonding $\left(d_{z^{2}}-5 \sigma\right)$ orbitals [14,15], which should be occupied for $\mathrm{Ni}$ and $\mathrm{Pt}$ and located close to the Fermi energy. So far, experimental studies of the valence bands of metals covered with $\mathrm{CO}$ found only indications for hybrid orbitals with a considerable admixture from CO $2 \pi$ character [7,24]. 
We report here the firs direct observation of an orbital close to the Fermi energy contributing to the chemisorption bond, but with no admixture from $\mathrm{CO} 2 \pi$ orbitals. Feature $D$ in the spectrum of $\mathrm{Ni}_{1}(\mathrm{CO})_{3}{ }^{-}$[Fig. 3(b)] exhibits a vibrational fin structure $\left(380 \pm 30 \mathrm{~cm}^{-1}\right)$ corresponding to the CO-metal vibration. Therefore, the orbital responsible for this feature contributes to the chemisorption bond. Feature $D$ does not exhibit a fin structure corresponding to the $\mathrm{CO}$ stretch vibration and we conclude that this orbital has no contribution from $\mathrm{CO} 2 \pi$ character. The only other possibility is an orbital with an admixture of CO $5 \sigma$ character. Since at binding energies close to the Fermi energy orbitals are expected to have a predominantly metal $d$ contribution, we assign feature $D$ tentatively to emission from a $\left(d_{z^{2}}-5 \sigma\right)$ hybrid orbital. A contribution from metal $s$ orbitals seems unlikely from the general similarity of the $\mathrm{Ni}_{n}(\mathrm{CO})_{m}{ }^{-}$and $\mathrm{Pt}_{n}(\mathrm{CO})_{m}{ }^{-}$ spectra. Because of the removal of an antibonding electron the CO-metal frequency should increase. However, in the fina state of the transition corresponding to feature $D$ there is still an electron occupying the CO $2 \pi$ orbital, which is antibonding with respect to the metal$\mathrm{CO}$ bond. Therefore, the measured frequency $\left(380 \mathrm{~cm}^{-1}\right)$ corresponds roughly to the one measured on a $\mathrm{Ni}$ surface $\left(400 \mathrm{~cm}^{-1}\right.$ [25]). Our observation of an occupied $d_{z^{2}}-5 \sigma$ hybrid orbital supports the results of Bauschlicher and Bagus [15], who predicted a nonbonding character of the $5 \sigma / d_{z^{2}}$ interaction for transition metals with ten or more valence electrons, because both the bonding and antibonding orbitals are occupied.

Summarizing this firs photoemission study of the reaction of $\mathrm{CO}$ with mass-separated $\mathrm{Ni}$ and Pt clusters, we found the formation of an electronic closed shell to be the reason for saturation. The extremely low frequencies of the $\mathrm{CO}$ stretch vibration in neutral $\mathrm{Ni}_{2}(\mathrm{CO})$ and $\mathrm{Ni}_{3}(\mathrm{CO})$ determined from the anion spectra indicate a stronger $\pi$ backdonation interaction for small particles in agreement with a recent determination of the binding energy of $\mathrm{CO}$ to a $\mathrm{Pt}_{3}$ cluster [23]. Finally, for $\mathrm{Ni}_{1}(\mathrm{CO})_{3}{ }^{-}$we observed a feature, which can tentatively be assigned to the antibonding $\left(d_{z^{2}}-5 \sigma\right)$ orbital supporting the assumption of a nonbonding $5 \sigma / d_{z^{2}}$ interaction for $\mathrm{Ni}[15]$.

[1] G. Blyholder, J. Phys. Chem. 68, 2772 (1964); see also N. Rösch and G. Pacchioni, in Clusters and Colloids, edited by G. Schmidt (Verlag Chemie, Weinheim, 1994), p. 5.

[2] J. W. Lauher, J. Am. Chem. Soc. 100, 5305 (1978).

[3] P. Fayet, M. J. McGlinchey, and L.H. Wöste, J. Am. Chem. Soc. 109, 1733 (1987), and references therein.

[4] P. A. Hintz and K. M. Ervin, J. Chem. Phys. 100, 5715 (1994), and references therein.
[5] P. A. Hintz and K. M. Ervin, J. Chem. Phys. 103, 7897 (1995).

[6] E. K. Parks, L. Zhu, J. Ho, and S. J. Riley, J. Chem. Phys. 100, 7206 (1994).

[7] N. V. Richardson and A. M. Bradshaw, Surf. Sci. 88, 255 (1979).

[8] C. de la Cruz and N. Sheppard, J. Mol. Struct. 224, 141 (1990).

[9] F. M. Hoffman, Surf. Sci. Rep. 3, 107 (1983).

[10] J. A. Timney, Inorg. Chem. 18, 2502 (1979).

[11] H. Handschuh, G. Ganteför, and W. Eberhardt, Rev. Sci. Instrum. 66, 3838 (1995).

[12] A. E. Stevens, C. S. Feigerle, and W. C. Lineberger, J. Am. Chem. Soc. 104, 5026 (1982).

[13] A. Nakajima, T. Taguwa, and K. Kaya, Chem. Phys. Lett. 221, 436 (1994).

[14] R. Hoffmann, Rev. Mod. Phys. 60, 601 (1988).

[15] C. W. Bauschlicher, Jr. and P. S. Bagus, J. Chem. Phys. 81, 5889 (1984).

[16] At photon energies close to threshold the photoemission cross section is larger for atomic $s$ and $p$ orbitals than for $d$ and $f$ ones. This cross section dependence [J. J. Yeh and I. Lindau, At. Data Nucl. Data Tables 32, 1 (1985)] is quite often used in solid state physics to assign emission features. In the case of $d$ and $f$ orbitals this can be justified because these orbitals are localized and not basically different from the ones of a free atom. For the strongly delocalized metal $s$ (or $\sigma$ ) and $p$ (or $\pi$ ) orbitals such an analogy is less valid. However, the CO $2 \pi$ orbitals are relatively localized, too, and the shape of a $\pi^{*}$ orbital is basically similar to two noninteracting $\mathrm{C}$ and $\mathrm{O}$ atomic $p$ orbitals.

[17] The feature at lowest BE in most cases corresponds to the transition from the electronic ground state of the anion into the neutral ground state of the cluster in the geometry of the anion. Therefore, we assign the vibrational frequencies $\left(A-B-C: 2020 \pm 60 \mathrm{~cm}^{-1} ; A-D-E\right.$ : $\left.425 \pm 25 \mathrm{~cm}^{-1}\right)$ in the spectrum of $\mathrm{Pt}_{3}(\mathrm{CO})_{6}{ }^{-}$[Figs. 2(d) and 3(a)] to modes of the neutral in its electronic ground state. We assume identical symmetries of the anion and neutral $\mathrm{Pt}_{3}(\mathrm{CO})_{6}{ }^{-}$, because the electron occupies an orbital derived from the CO $2 \pi$ orbitals [18].

[18] D. W. Bullett, Chem. Phys. Lett. 115, 450 (1985).

[19] W. D. Mieher, L. J. Whitman, and W. Ho, J. Chem. Phys. 91, 3228 (1989), and references therein.

[20] M. E. Davila et al., Surf. Sci. 311, 337 (1994).

[21] G. Ganteför, G. Schulze Icking-Konert, H. Handschuh, and W. Eberhardt (to be published).

[22] L. Surnev, Z. Xu, and J. T. Yates, Jr., Surf. Sci. 201, 1 (1988).

[23] A. Grushow and K. M. Ervin, J. Am. Chem. Soc. 117, 11612 (1995).

[24] H.-J. Freund and H. Kuhlenbeck, in Applications of Synchrotron Radiation, edited by W. Eberhardt, Springer Series in Surface Science Vol. 35 (Springer-Verlag, Berlin, 1995), p. 9, and references therein.

[25] S. Anderson, Solid State Commun. 21, 75 (1979). 